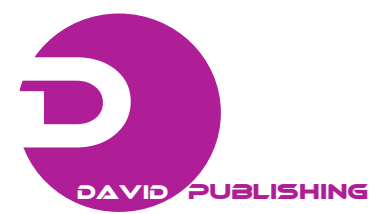

\title{
Steel Valve Plate Grinding
}

\author{
Dijana Nadarevic and Mirko Sokovic \\ Faculty of Mechanical Engineering, University of Ljubljana, Ljubljana SI-1000, Slovenia
}

Received: January 28, 2013 / Accepted: March 1, 2013 / Published: April 25, 2013.

\begin{abstract}
In the optimization of the process of steel valve plate grinding, it is necessary to take into consideration different processing parameters. This paper is going to describe the production of a steel valve plate with the focus on flat-grinding. It is also going to present the method of choosing grinding wheels and parameters, as well as their impact on the processing quality parameters (roughness, surface flatness and seat width). The optimization of the grinding process ensures acceptable quality of the final product applying the smallest possible changes in parameters at non-uniform quality levels of the tools. Here we have to make sure that the machining process is acceptable in terms of processing cost as well. We are going to use the G-factor as the efficiency indicator for different tools.
\end{abstract}

Key words: Valve plates, flat-grinding, G-factor.

\section{Introduction}

At the beginning of the planning of the grinding process, it is first of all necessary to collect the data about the work piece, the available machine, processing operations, types of grinding wheels, etc. Based on these starting points (input parameters) and on the already known output parameters (the surface flatness and roughness, the seat width, the process costs, etc.), it is possible to optimize the flat-grinding process to such degree that the quality of the product is acceptable even at minimum production costs.

In continuation, the process of the high-serial production of a steel valve plate, a part of a household compressor, is described. A particularity of such valve plate is in the difficulty of production both for the geometric shape and its low hardness.

Due to the above mentioned difficulties, the further focus is set on the choice of processing parameters and grinding tools necessary for the efficient grinding.

The criterion by which it has been decided whether

Dijana Nadarevic, Ph.D. student, research fields: process technology, quality.

Corresponding author: Mirko Sokovic, professor, Ph.D., research fields: production technology, quality management. E-mail: mirko.sokovic@fs.uni-lj.si. the chosen parameters are suitable or not is called the G-factor, and it is calculated as a ratio between the ground material of the work piece and the tools (Eqs. (2)-(4)). Its meaning would be additionally underlined in a completely automated machining process.

In the choice of processing parameters and tools, as well as in the process analyses, different methods have been used: design of experiment (DOE) for the choice of input-output parameters, and the analysis of variance for the comparison of critical populations. The paper mentions only the final results of the above mentioned analyses, which are based on previous researches [1].

The experimental results prove that it is possible to select optimal tools and grinding parameters to achieve the best quality of the final product. The paper is organized in the following way: Section 2 presents the product description; section 3 describes the steel valve plate processing; section 4 presents the way grinding parameters are chosen; section 5 presents the selection of optimal grinding wheels; section 6 presents research results and analyses; section 7 offers conclusions and possibilities for future researching. 


\section{Valve Plate Presentation}

Fig. 1 presents a valve plate made of steel St20, dimensions $56 \times 60 \times 2.1 \mathrm{~mm}^{3}$.

Low hardness of 117 to $170 \mathrm{HV}$ is typical for a steel valve plate. The seat of raw material is rather inadequate for the automatic feeding, a consequence of the pressed material of the seat. Due to the rapid corrosion of the raw material, a supplier has to protect the material from corrosion, which causes plate gluing and makes the automating feeding difficult.

The product designing is complex both for the possibility of the primary burr (resulting from the stamping process, especially at the intersection of two smaller radii) and for the secondary burr (resulting from the grinding process).

The following section discusses only those grinding wheels and parameters which have given acceptable results in terms of secondary burrs.

\section{Production of Steel Valve Plate}

After the verification of the raw material (stamped valve plate) in the "Incoming inspection" section, the plates are transported to the store for the flat-grinding operation. The grinding process is performed on the machine for two-sided flat-grinding: Diskus Werke AG, Germany [1].

After that the grinding operator transports the valve plates in boxes to the brushing operation-Vobhag, Switzerland. The plates travel from the load via a conveyer belt to the magnet plates of the machine and are brushed first from one side at the first station (Rotex 1, Switzerland) - a station with three brushes $\varnothing 150 \mathrm{~mm}$ ) and then they are rotated and brushed on the other side at the second station (Rotex 2, Switzerland). The aim of this operation is primarily to remove the secondary, as well as the primary, burr from the valve plates and to even the sharp edges, especially the ones on the seats of the surface of a valve. After the brushing process the valve plate is rotated by $90^{\circ}$ (vertical position) and is transported to the washing operation.

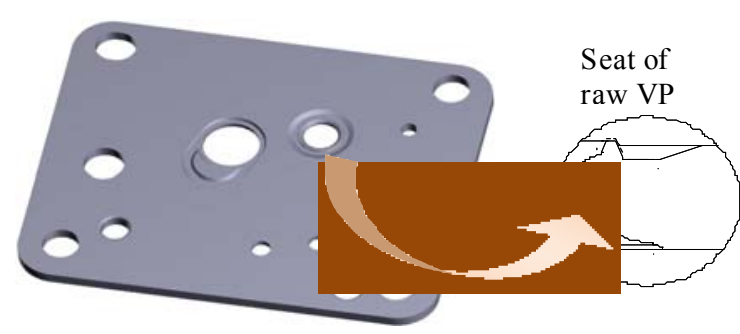

Fig. 1 Steel valve plate.

After the washing, the operator first visually inspects the valve plates, then stacks them in cardboard boxes and puts them onto the shelves for the finished valve plates. The last operation includes the valve system assembly.

\subsection{Steel Valve Plate Grinding}

Flat-grinding is a process of material subtraction, in which a high accuracy and a high surface quality can be achieved. The main movement is performed by two grinding wheels rotating very fast [3]. In this case, the feeding is performed by a wheel or a transport plate with planets, as shown in Fig. 2.

Double-sided flat-surface grinding is a process in which the material is removed from the workpiece from both sides simultaneously [4]. The uniformity of the removal of material is guaranteed by setting optimal grinding parameters.

The grinding process itself is conducted so that the operator manually puts the valve plates onto the planets of the transport plate which then carries them between the grinding wheels and the grinding process begins. During the work the operator monitors the thickness of the valve plates (consequently also the seat width), the roughness and the flatness of the surface. On the basis of these control variables he decides about his further action: changing the thickness of the raw valve plate, more frequent wheel dressing, changing the speed of the wheels...

\section{Choice of Steel Valve Plate Parameters}

In the selection of optimal parameters in flat-grinding, the major part of the problems arose from the instability of the quality of grinding wheels. Table 1 


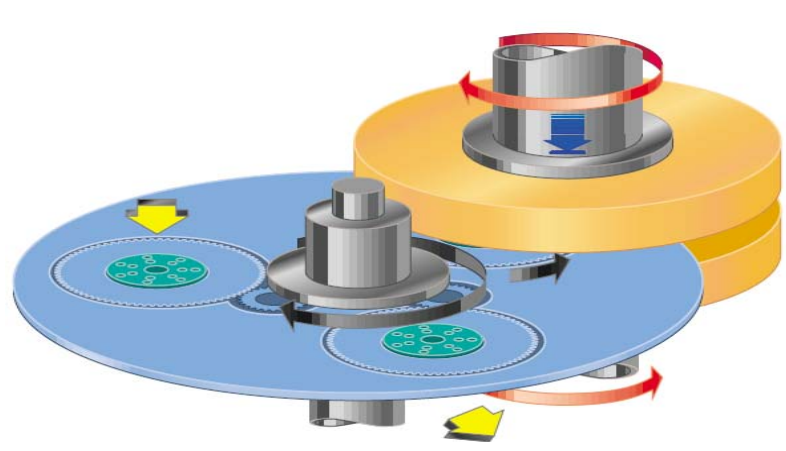

Fig. 2 Movement of grinding wheels and feeding plate.

Table 1 Unchanging grinding parameters.

\begin{tabular}{ll}
\hline Grinding parameters & Values \\
\hline Transport plate grinding speed & $8000 \mathrm{rpm}$ \\
Planet rotation speed & $80 \mathrm{rpm}$ \\
Planet rotation direction & To the right (1) \\
Partial shift of the z-axis & $0.040 \mathrm{~mm}$ \\
Spindle rotation & counter direction \\
\hline
\end{tabular}

shows the parameters that remain mainly unchanged despite the unstable quality of grinding wheels. Besides the above given unvarying parameters we also have the adjustable parameters, such as the wheel rotation speed, the thickness of the raw material, the number of the transitions in dressing, etc. The speed of the feeding plate has no significant influence on the product quality.

The process analyses have shown that the wheel rotation speed has a big importance in the quality of grinding.

The optimal value is determined and it is also the maximum value permitted by the machine $\left(32 \mathrm{~m} \mathrm{~s}^{-1}\right)$. According to the machine manufacturer it is possible to specify the optimal planet rotation speed (approximate values) from the speed ratio, as shown in Table 2.

Speed ratio can be written as Eq. (1):

$$
q_{s}=60 \cdot \frac{v_{s}}{v_{w}}=\frac{n_{s} \cdot d_{s}}{n_{w} \cdot d_{w}}
$$

where $n_{s}$ is the number of rotations of grinding wheels; $v_{s}$ is the peripheral speed of wheels in $\mathrm{m} \mathrm{s}^{-1} ; n_{w}$ is the number of rotations of the workpiece; $v_{w}$ is the peripheral speed in $\mathrm{m} \mathrm{s}^{-1}$ of the work piece (planets with the diameter of Ø230 mm).
Table 2 Recommended values of speed ratios.

\begin{tabular}{ll}
\hline Process & Indicator-orientation values $q_{s}$ \\
\hline Rough grinding & $40-60$ \\
Fine grinding & $25-40$ \\
\hline
\end{tabular}

Other important processing parameters include the dressing parameters. Namely, the quality of the finished product (especially the flatness of the valve plate) depends on the quality of dressing. These parameters are: the speed of dressing wheels, the number of dressing transitions and the thickness of the raw material during the dressing.

Based on the performed tests, it was found that the quality parameters were satisfied (the roughness and the flatness of the surface) in terms of the material removal of $0.02 \mathrm{~mm}$ and at five dressing wheel transitions.

The optimal processing parameters for flat-grinding have been shown in Table 3 [1].

The ratio of $32 / 28 \mathrm{~m} \mathrm{~s}^{-1}$ has been taken for the rotation speed of grinding wheels since this combination ensures a uniform material removal on both sides of a workpiece.

Depending on the speed of the grinding wheel wear, the frequency of the manual shift of the bottom grinding wheel is determined.

\section{Choice of Wheels for the Flat Grinding of Valve Plates}

The suppliers of grinding wheels can only hardly ensure equal resistance of grinding wheels, even though the process of their production is the same for each pair with the same specifications.

Grain size is selected on the basis of the required surface quality of the workpiece [5]. For the roughness of $R a<0.3 \mu \mathrm{m}$, recommended by the producer, we can choose the medium granulation of grinding grains. The tests have shown that in the area of fine granulation better results have been achieved, especially in the case of smaller thickness of the raw material. Some producers also use double marks for granularity, for example 150/2. This code means that one third of the 
Table 3 The optimal valve plate grinding parameters.

\begin{tabular}{ll}
\hline Grinding parameters & Values \\
\hline Grinding wheel rotation speed & $32 / 28 \mathrm{~m} \mathrm{~s}^{-1}$ \\
Thickness of raw material & $0.1 \mathrm{~mm}$ \\
Number of dressing transitions & 5 \\
Dressing frequency & $1 \mathrm{~h}$ \\
\hline
\end{tabular}

grain mixture has the granulation of 150 , one third has one level lower granulation (120) and the last third of the mixture has one level higher granulation (180).

In the choice of hardness and structure of wheels, we considered the advice that it is necessary to choose harder grinding wheels to grind softer material. The problem occurred in the determination of the structure of wheels. In a more open structure we had problems with scratches on the valve plate. Selecting the wheels with lower hardness and more closed structure we the grinding wheels started "levelling", what means that the grains were wearing out and were not pushing, which resulted in the loss of the cutting capacity of grinding wheels.

Experiments have shown that the medium hard and hard wheels are the most suitable in grinding a steel valve plate.

Ceramic bounded grinding wheels are normally used at the operating speed of $40 \mathrm{~m} \mathrm{~s}^{-1}$, they maintain the form very well and are useful for all types of grinding (from very rough to the finest).

Bakelite has proved to be a better solution for the burr, but there were many problems with so-called "watering of wheels" (change of the characteristics of wheels after a certain period of time). This phenomenon is observed especially in the period when the space is unheated or in case of a longer machine failure.

It should be stressed that each analysis had to be performed for each supplier separately since the grinding wheels with the same marks are quite different at different suppliers [6].

Experiments have shown that we get similar results with the following grinding wheels:

- 58A150/1R8B25;

- 52A150/2L5B22;
- 50A180J5AV217.

That means that we are talking about a mixture of the corundum of 150, 120 and 180 granulation (depending on the specified ratio), the medium $(L)$ or high $(R)$ hardness, and the closed structure with the Bakelite binder (exceptionally ceramic).

A more detailed analysis has been made for Bakelite wheels since they are used the most frequently. The results of Bakelite grinding wheels with the granulation of 120 and 150 (mixture), hardness $R$ (hard) and closed structure (8) have been compared to the grinding wheels with the mixture of grains with the granulation of 120, 150 and 180, medium hardness $(L)$ and closed structure.

The dressing of grinding wheels have been performed in all cases using the diamond dressing tool in dimensions of $\varnothing 120 \times 20 \times 51 \mathrm{~mm}^{3}$, mark 119D126-30MB02.

\section{Analysis of the Results}

The analysis is based on the optimal process parameters (Table 3) for grinding wheels of two different suppliers (supplier A and supplier B).

As a criterion for the suitability of individual tools, the G-factor has been chosen and it represents the ratio between the volume of raw materials and the grinding wheel wear, as shown in Eq. (2):

$$
G=\frac{V_{w}}{V_{s}}
$$

where $V_{s}$ is the volume of the grinding wheel wear and $V_{w}$ is the volume of the raw material.

The volume of the grinding wheel wear is calculated using Eq. (3):

$$
V_{s}=\frac{\pi \cdot\left(D^{2}-d^{2}\right)}{4}
$$

where $D$ is the outer diameter of wheels; $d$ is the inner diameter of wheels.

The volume of the raw materials can be written as Eq. (4):

$$
V_{w}=n \cdot l \cdot h \cdot \delta
$$

where $n$ is the number of valve plates ground by one pair of grinding wheels; $l$ is the length of the workpiece; 
$h$ is the width of the workpiece; $\delta$ is the thickness of the raw material.

According to the values obtained for both parameters ( $C p$ and $C p k>1.33)$, we can claim that the process is capable.

The lower tolerance limit is presented by the value which can be reached in a real process. In case the value 0 is taken as the lower limit the process centring is worse, which in our case can also be accepted?

In continuation we are going to compare the performance of two different grinding wheels $(\mathrm{GW})$ :

- GW 1 = 58A150/1R8B25 (supplier A);

- $\mathrm{GW} 2$ = 52A150/2L5B22 (supplier B).

Table 4 presents an example of the efficiency of two wheels with different features.

Before we check the value of G-factor, it is necessary to verify the capability of the valve plate grinding process for selected grinding tools and parameters. The process capability index $(C p, C p k)$ has been chosen as the indicator of quality.

Fig. 3 shows the process capability index for both quality parameters: the flatness and the roughness of a valve plate.

G-factor for hard wheels (1) is a little higher. This result was expected since the softer wheels (2) require more frequent measure corrections (the removal of the raw material). Due to the manual adjustment of the

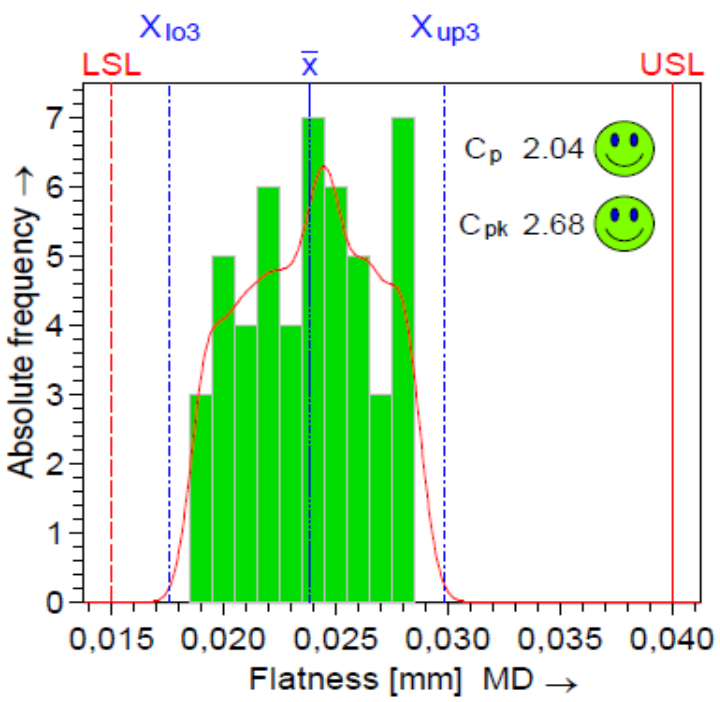

Fig. 3 Process capability indexes.
Table 4 Comparison of G-factors of grinding wheels (GW) taken from different suppliers.

\begin{tabular}{lll}
\hline Type of grinding wheel & $\begin{array}{l}\text { GW 1 } \\
\text { (supp. A) }\end{array}$ & $\begin{array}{l}\text { GW 2 } \\
\text { (supp. B) }\end{array}$ \\
\hline Outer diameter of GW $D(\mathrm{~mm})$ & 456 & 455 \\
Inner diameter of GW $d(\mathrm{~mm})$ & 220 & 220 \\
GW height $T(\mathrm{~mm})$ & 45 & 45 \\
Length $x$ width of workpiece $l(\mathrm{~mm})$ & $60 \times 56$ & $60 \times 56$ \\
Raw material thickness $\delta(\mathrm{mm})$ & $0.1+0.03$ & $0.1+0.03$ \\
Nr. of valve plates $n($ pieces $)$ & 40446 & 32175 \\
Volume of GW $V_{s}\left(\mathrm{~mm}^{3}\right)$ & 5635509 & 5603428 \\
Volume of raw valve plate $V_{w}\left(\mathrm{~mm}^{3}\right)$ & 10569888 & 10810800 \\
G-factor & $2.41-3.13$ & $1.93-2.51$ \\
\hline
\end{tabular}

quantity of subtraction it is not possible to specify the G-factor exactly. However, we can define the area in which it is located.

\section{Conclusions}

Due to the unequal quality of grinding wheels, the major part of tests performed includes the choice of the optimal type of wheels.

Since the requirements included lower roughness of the processed surface at higher material removal certain difficulties have been encountered. An incorrect choice of grinding wheels has also caused an increased wheel wear. This resulted in smaller cutting capability and in fast wheel dressing, which means that the processing costs were higher.

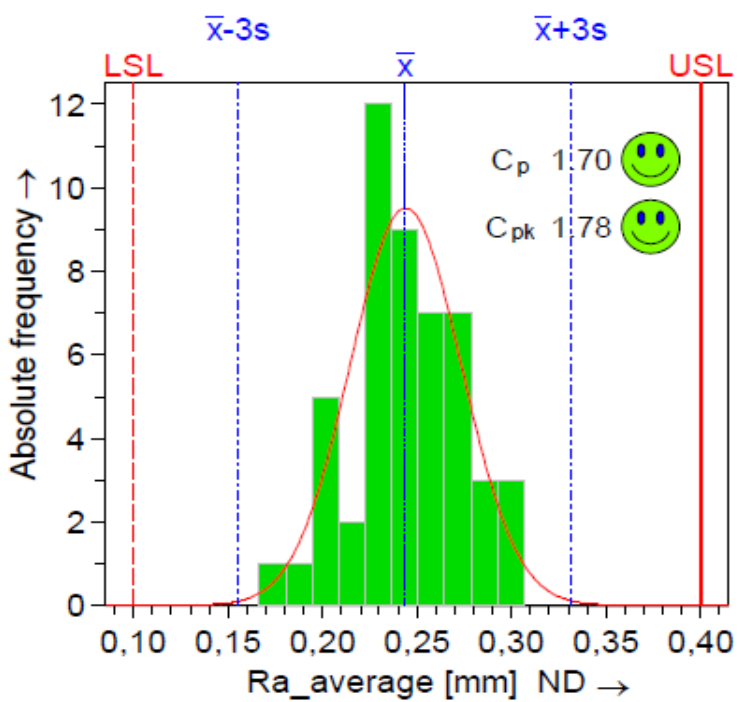


The automation in setting the material removal, the possibility to choose higher speed of grinding wheels and the provision of stability in the quality of wheels have a great influence on both the quality and the cost optimization of the steel valve plate grinding process.

In case of satisfaction of the mentioned requirements the G-factor would improve, as well, mainly due to the decrease in the frequency of the grinding wheel dressing.

\section{References}

[1] D. Nadarević, M. Soković, Planning the steel valve plate grinding process, in: 6th Int. Conf. ICQME'2011, Tivat, Montenegro, 2011.

[2] Double Face Grinding Machines, Catalogue, Diskus Werke AG, Germany, 2001.

[3] http://diskus-werke.dvs-gruppe.com/index.php?.

[4] Technische Dokumentation: Betriebanleitung/Operating Instructions, Diskus Werke AG, Germany, 2001.

[5] S. Malkin, G. Changsheng, Grinding Technology, United Technologies Research Center, New York, 2008.

[6] Grinding Instructions, Internal Manual, Swaty d.d., Factory for Grinding Wheel Production, Slovenia, 2010. 